PROCEEDINGS OF THE

AMERICAN MATHEMATICAL SOCIETY

Volume 139, Number 7, July 2011, Pages 2317-2325

S 0002-9939(2010)10654-8

Article electronically published on December 16, 2010

\title{
SOME REMARKS ON SYMPLECTIC INJECTIVE STABILITY
}

\author{
RABEYA BASU, PRATYUSHA CHATTOPADHYAY, AND RAVI A. RAO \\ (Communicated by Martin Lorenz)
}

\begin{abstract}
It is shown that if $A$ is an affine algebra of odd dimension $d$ over an infinite field of cohomological dimension at most one, with $(d+1) ! A=A$, and with $4 \mid(d-1)$, then $\operatorname{Um}_{d+1}(A)=e_{1} \operatorname{Sp}_{d+1}(A)$. As a consequence it is shown that if $A$ is a non-singular affine algebra of dimension $d$ over an infinite field of cohomological dimension at most one, and $d ! A=A$, and $4 \mid d$, then $\operatorname{Sp}_{d}(A) \cap \mathrm{ESp}_{d+2}(A)=\mathrm{ESp}_{d}(A)$. This result is a partial analogue for evendimensional algebras of the one obtained by Basu and Rao for odd-dimensional algebras earlier.
\end{abstract}

\section{INTRODUCTION}

The rings $R$ we work with are always commutative with 1 . In 2 Bass-MilnorSerre began the study of the stabilization for the linear groups $\mathrm{GL}_{n}(R) / \mathrm{E}_{n}(R)$, for $n \geq 3$. They showed that the natural map $\mathrm{GL}_{n}(R) / \mathrm{E}_{n}(R) \longrightarrow \mathrm{GL}_{n+1}(R) / \mathrm{E}_{n+1}(R)$ is an isomorphism for $n \geq d+3$, where $d$ is the Jacobson-Krull dimension of $R$ (i.e. the dimension of its maximum spectrum). In [3], $\S 11$, Bass conjectured that $K_{1}(R)=\mathrm{GL}_{d+2}(R) / \mathrm{E}_{d+2}(R)$, where $d$ is the Jacobson-Krull dimension. In [23], L.N. Vaserstein proved this conjecture for an algebra $A$, which is finite as a module over a commutative ring $R$ and whose spectrum of maximal ideals is a noetherian space of dimension $d$.

In [14] R.A. Rao and W. van der Kallen showed that if $A$ is a non-singular affine algebra of dimension $d>1$ over a field of cohomological dimension at most one, then the natural map $\mathrm{SL}_{n}(A) / \mathrm{E}_{n}(A) \longrightarrow \mathrm{SL}_{n+1}(A) / \mathrm{E}_{n+1}(A)$ is injective for $n \geq d+1$, if $(d+1) ! A=A$. The expectation that the injective stability would improve over these special rings was motivated by the famous theorem of A. Suslin (in [22]) who showed that stably free projective modules of rank $d$ over such rings were free, improving the earlier estimate of $(d+1)$ of $\mathrm{H}$. Bass over general commutative rings.

L.N. Vaserstein in 24] pursued the question of injective stability for the other classical groups: the symplectic groups $\operatorname{Sp}_{2 n}(R) / \operatorname{ESp}_{2 n}(R)$, and the orthogonal groups $\mathrm{O}_{2 n}(R) / \mathrm{EO}_{2 n}(R)$. He showed that the natural maps $\operatorname{Sp}_{2 n}(R) / \mathrm{ESp}_{2 n}(R) \rightarrow$ $\mathrm{Sp}_{2 n+2}(R) / \mathrm{ESp}_{2 n+2}(R)$ and $\mathrm{O}_{2 n}(R) / \mathrm{EO}_{2 n}(R) \rightarrow \mathrm{O}_{2 n+2}(R) / \mathrm{EO}_{2 n+2}(R)$ are bijective for $n \geq(2 d+4)$, where $d$ is the stable dimension of $R$.

In [4] it was shown that if $A$ is a non-singular affine algebra of dimension $d$ over a field of cohomological dimension at most one, then in the symplectic situation the

Received by the editors January 9, 2010 and, in revised form, January 12, 2010; June 9, 2010; and June 18, 2010.

2000 Mathematics Subject Classification. Primary 13C10, 13H05, 19B14, 19B99, 55R50.

Key words and phrases. Unimodular rows, elementary symplectic group. 
above map is injective for $n \geq d+1$. In ([ $[6$, Corollary 6.8) the above result of 4 ] is re-proved via a relative version of a lemma of L.N. Vaserstein in ([17, Lemma 5.5). Moreover, it is shown that if $A$ is a non-singular affine algebra over a finite field or its algebraic closure, of even dimension $d \geq 4$, of characteristic $\neq 2$, then the above map is injective for $n \geq d$.

In this paper we extend the results in the symplectic case, and our main result is the following:

Theorem 1. Let $A$ be an affine algebra of even dimension $d$ over a field $k$ of cohomological dimension at most one. If $(d+1) ! A=A$ and $4 \mid d$, then a $\sigma \in S p_{d}(A) \cap$ $E_{S p_{d+2}}(A)$ is symplectic homotopic to the identity. In particular, if $A$ is nonsingular, then the natural map

$$
\frac{\operatorname{Sp}_{2 n}(R)}{\operatorname{ESp}_{2 n}(R)} \rightarrow \frac{\operatorname{Sp}_{2 n+2}(R)}{\operatorname{ESp}_{2 n+2}(R)}
$$

is injective for $2 n \geq \max \{4, d\}$.

The improvement in the bound is mainly due to the use of Suslin matrices, as done in [15], the emphasis here being to use the sizes when the Suslin matrices are symplectic. This explains the need for the condition on the dimension as the Suslin matrices exhibit four different characteristics depending on the size of the row. Here are a few more comments on the proof.

For an affine algebra of dimension $d$ over a field of cohomological dimension at most one, A. Suslin in 22 has shown that $\operatorname{Um}_{d+1}(A)=e_{1} \mathrm{SL}_{d+1}(A)$. As a consequence of our argument one can improve this result in some cases and show the following.

Theorem 2. Let $A$ be an affine algebra of odd dimension $d \geq 5$ over a field of cohomological dimension at most one. Assume that $d ! A=A$. If $4 \mid(d-1)$, then $U m_{d+1}(A)=e_{1} S p_{d+1}(A)$.

Theorem 1 is a consequence of Theorem 2 .

The study of injective stabilization is deeply connected to the question of whether stably free modules are free. We refer the reader to $([15, \S 2)$, where this connection has been detailed.

Suslin's expectation in 21] is that a stably free module of rank $d-1$ over an affine algebra over an algebraically closed field is free. J. Fasel has recently proved a remarkable result in [8] that this is true over smooth threefolds. In view of the connection mentioned above between freeness of projective modules defined by a unimodular row and stably elementary matrices, we expect that the correct estimate will be $2 n \geq \max \{4, d-1\}$ for non-singular algebras $A$ (with $d ! A=A$ ) over a field of cohomological dimension one. (If this is shown, then one can show that Suslin's conjecture holds for affine algebras of dimension $d \equiv 1$ modulo (4).)

J. Fasel in ( 8 , Corollary 3.3) has shown that if $A$ is a smooth affine algebra of dimension three over a field of cohomological dimension at most one and $3 ! A=A$, then $\mathrm{Um}_{4}(A) / \mathrm{E}_{4}(A) \mathrm{Sp}_{4}(A)=0$, provided also that -1 is a square in $A$. This raises the question whether $\operatorname{Um}_{d+1}(R)=e_{1} \operatorname{Sp}_{d+1}(R)$ when $d ! A=A$ and $2 \mid(d-1)$ over an affine algebra of dimension $d$ over a field of cohomological dimension at most one. Our method does not apply unless $4 \mid(d-1)$ and $(d+1) ! A=A$.

This is the first occasion that the form of the Suslin matrices has been used to get a positive feedback on the question of injective stability in the case of the 
symplectic groups. On an earlier occasion in [16 the theory of Suslin matrices expounded by Jose-Rao in [9, 10] has been used to show that the injective stability bounds do not improve in general for the orthogonal groups; see (5], Corollary 2.7), where an example of an affine algebra of dimension 3 over a field of cohomological dimension one is given for which the map between orthogonal quotients is bijective for $2 n \geq 8$ but is not injective for $2 n=6$.

\section{Preliminaries}

2.1. A relative version of a lemma of L.N. Vaserstein. We begin with some preliminary definitions.

A row $v=\left(v_{1}, \ldots, v_{n}\right) \in R^{n}$ is said to be unimodular if there are elements $w_{1}, \ldots, w_{n}$ in $R$ such that $v_{1} w_{1}+\cdots+v_{n} w_{n}=1 . \operatorname{Um}_{n}(R)$ will denote the set of all unimodular rows $v \in R^{n}$. Let $I$ be an ideal in $R$. We denote by $\operatorname{Um}_{n}(R, I)$ the set of all unimodular rows of length $n$ which are congruent to $e_{1}=(1,0, \ldots, 0)$ modulo I. (If $I=R$, then $\operatorname{Um}_{n}(R, I)$ is $\operatorname{Um}_{n}(R)$.)

The group $\mathrm{GL}_{n}(R)$ of invertible matrices acts on $R^{n}$ in a natural way: $v \mapsto v \sigma$, if $v \in R^{n}, \sigma \in \mathrm{GL}_{n}(R)$. This map preserves $\operatorname{Um}_{n}(R)$, so $\mathrm{GL}_{n}(R)$ acts on $\mathrm{Um}_{n}(R)$. Note that any subgroup $G$ of $\mathrm{GL}_{n}(R)$ also acts on $\operatorname{Um}_{n}(R)$. If $v, w \in \operatorname{Um}_{n}(R)$, we denote $v \sim_{G} w$ or $v \in w G$ if there is a $g \in G$ such that $v=w g$.

Let $\mathrm{E}_{n}(R)$ denote the subgroup of $\mathrm{SL}_{n}(R)$ consisting of all elementary matrices, i.e. those matrices which are a finite product of the elementary generators $\mathrm{E}_{i j}(\lambda)=$ $\mathrm{I}_{n}+\lambda e_{i j}, 1 \leq i \neq j \leq n, \lambda \in R$, where $e_{i j} \in \mathrm{M}_{n}(R)$ has only one non-zero entry 1 in its $(i, j)$-th position.

Definition 2.1 (The Relative Groups $\mathrm{E}_{n}(I), \mathrm{E}_{n}(R, I)$ ). Let $I$ be an ideal of $R$. The relative elementary group $\mathrm{E}_{n}(I)$ is the subgroup of $\mathrm{E}_{n}(R)$ generated as a group by the elements $\mathrm{E}_{i j}(x), x \in I, 1 \leq i \neq j \leq n$.

The relative elementary group $\mathrm{E}_{n}(R, I)$ is the normal closure of $\mathrm{E}_{n}(I)$ in $\mathrm{E}_{n}(R)$.

An important theorem of A.A. Suslin in ([19], Corollary 1.4) is that $\mathrm{E}_{n}(R, I)$ is a normal subgroup of $\mathrm{GL}_{n}(R)$, for $n \geq 3$.

The following is a special case of Bass' stabilization results in ([1], Chapter 4, Theorem 3.4, pg. 183):

Proposition 2.2. Let $A$ be a commutative ring whose maximum spectrum has dimension $\leq d$, and let $I$ be an ideal of $A$. Then $\operatorname{Um}_{r}(A, I)=e_{1} E_{r}(A, I)$, for $r \geq \max \{3, d+2\}$.

Definition 2.3 (Symplectic Group $\operatorname{Sp}_{2 n}(R)$ ). This is the isotropy group of the standard symplectic form $\psi_{n}=\sum_{i=1}^{n} e_{2 i-1,2 i}-\sum_{i=1}^{n} e_{2 i, 2 i-1}$. In other words, $\operatorname{Sp}_{2 n}(R)=\left\{\alpha \in \mathrm{GL}_{2 n}(R) \mid \alpha^{t} \psi_{n} \alpha=\psi_{n}\right\}$.

Let $\sigma$ denote the permutation of the natural numbers given by $\sigma(2 i)=2 i-1$ and $\sigma(2 i-1)=2 i$.

Definition 2.4 (Elementary Symplectic Group $\operatorname{ESp}_{2 n}(R)$ ). We define for $z \in R$, $1 \leq i \neq j \leq 2 n$,

$$
s e_{i j}(z)= \begin{cases}1_{2 n}+z e_{i j} & \text { if } i=\sigma(j), \\ 1_{2 n}+z e_{i j}-(-1)^{i+j} z e_{\sigma(j) \sigma(i)} & \text { if } i \neq \sigma(j) \text { and } i<j .\end{cases}
$$


(It is easy to check that all these generators belong to $\operatorname{Sp}_{2 n}(R)$.) We call them the elementary symplectic matrices over $R$, and the subgroup of $\operatorname{Sp}_{2 n}(R)$ generated by them is called the elementary symplectic group $\operatorname{ESp}_{2 n}(R)$.

Definition 2.5 (The Relative Group $\operatorname{ESp}_{2 n}(I), \operatorname{ESp}_{2 n}(R, I)$ ). Let $I$ be an ideal of $R$. The relative elementary group $\operatorname{ESp}_{2 n}(I)$ is the subgroup of $\mathrm{ESp}_{2 n}(R)$ generated as a group by the elements $s e_{i j}(x), x \in I$ and $1 \leq i \neq j \leq 2 n$.

The relative elementary group $\mathrm{ESp}_{2 n}(R, I)$ is the normal closure of $\mathrm{ESp}_{2 n}(I)$ in $\operatorname{ESp}_{2 n}(R)$.

We now recall a well-known lemma of L.N. Vaserstein:

Lemma 2.6 ([17, Lemma 5.5]). For any natural number $n \geq 2$ and any alternating matrix $\varphi$ from $\mathrm{GL}_{2 n}(R)$, we have

$$
e_{1}\left(\mathrm{E}_{2 n}(R)\right)=e_{1}\left(\mathrm{E}_{2 n}(R) \cap \operatorname{Sp}_{\varphi}(R)\right)
$$

where

$$
\left.\operatorname{Sp}_{\varphi}(R)\right)=\left\{\alpha \in \mathrm{SL}_{2 n}(R) \mid \alpha^{t} \varphi \alpha=\varphi\right\} .
$$

In this special case when $\varphi=\psi_{r}$ the proof is much easier to establish. In this case his proof shows the following:

Lemma 2.7. For any natural number $n \geq 2, e_{1} \mathrm{E}_{2 n}(R)=e_{1} \operatorname{ESp}_{2 n}(R)$.

The relative version of Lemma 2.7 was established in [6]

Theorem 2.8 ([6, Theorem 5.5]). Let $I$ be an ideal of $R$ and $2 R=R$. Let $v \in$ $\operatorname{Um}_{2 n}(R, I)$. Then $v \mathrm{E}_{2 n}(R, I)=v \operatorname{ESp}_{2 n}(R, I)$, for $n \geq 2$.

Remark 2.9. One can similarly define the groups $\operatorname{ESp}_{\varphi}(R), \operatorname{ESp}_{\varphi}(R, I)$, w.r.t. an ideal $I$ of $R$, for any alternating matrix $\varphi \in \mathrm{SL}_{2 n}(\mathbb{Z})$, which is given by a (signed) permutation matrix (i.e. which is a permutation matrix if you ignore the signs). We have all the above results (Lemma 2.6, Lemma 2.7, Theorem 2.8) even in this situation. In the sequel we will be using these results with $\varphi=J_{r}$, a special alternating matrix defined by A. Suslin. (See Subsection 2.2 below for the definition of $J_{r}$.)

We next recall the role of the excision algebra (introduced by W. van der Kallen in (14], Proposition 3.3) to convert questions of actions of relative groups to actions of absolute groups. We abstract the proof of ([14], Proposition 3.3) as:

Lemma 2.10. Let $v \in \operatorname{Um}_{r}(A), r \geq 3$, with $v \equiv e_{1}$ modulo $(t)$, for some $t \in A$. Let $v=e_{1}+t w$, for $w \in A^{r}$. Let $B=A[Z] /\left(Z^{2}-t Z\right)$. Let $v^{*}=e_{1}+Z w$. Then its image in $B^{r}$ is unimodular. Moreover, if it is completable (to a (symplectic) matrix of determinant one) over $B$, then $v$ is completable (to a (symplectic) matrix of determinant one) over $A$ which is congruent to the identity modulo $(t)$.

$B$ is called the excision algebra w.r.t. $(t)$.

We now recall a few more results which will be needed in the sequel.

2.2. The Suslin matrix. Given two vectors $v, w \in R^{r+1}$, A.A. Suslin in ([18, $\left.\S 5\right)$ gave an inductive process to construct the (Suslin) matrix $S_{r}(v, w)$. We recall this process: Let $v=\left(a_{0}, v_{1}\right), w=\left(b_{0}, w_{1}\right)$, where $a_{0}, b_{0} \in R$ and $v_{1}, w_{1} \in \mathrm{M}_{1 r}(R)$. Set $S_{0}(v, w)=a_{0}$, and set

$$
S_{r}(v, w)=\left(\begin{array}{cc}
a_{0} I_{2^{r-1}} & S_{r-1}\left(v_{1}, w_{1}\right) \\
-S_{r-1}\left(w_{1}, v_{1}\right)^{T} & b_{0} I_{2^{r-1}}
\end{array}\right) .
$$


In (18], Lemma 5.1) it is noted that $S_{r}(v, w) S_{r}(w, v)^{T}=\left(v \cdot w^{T}\right) I_{2^{r}}=$ $S_{r}(w, v)^{T} S_{r}(v, w)$, and that $\operatorname{det} S_{r}(v, w)=\left(v \cdot w^{T}\right)^{2^{r-1}}$, for $r \geq 1$.

A. Suslin introduced these matrices and showed that a unimodular row of the form $\left(a_{0}, a_{1}, a_{2}^{2}, \ldots, a_{r}^{r}\right)$ (which is the same as $\chi_{r !}([v])$, for $v=\left(a_{0}, a_{1}, \ldots, a_{r}\right)$ in the notation of L. Vaserstein in [25]) can be completed to an invertible matrix. In fact, in (20, Proposition 2.2, Corollary 2.5) it is shown that there is a $\beta_{r}(v, w)$ with $\left[\beta_{r}(v, w)\right]=\left[S_{r}(v, w)\right]$ in $K_{1}(A)$ whose first row is $\chi_{r !}([v])$. In ([15], Corollary 4.3 (iii)) it is shown that if $v, w \in \operatorname{Um}_{r+1}(R, I)$, then we may assume that $\beta_{r}(v, w) \equiv$ $I_{r+1}$ modulo $I$.

A.A. Suslin then describes a sequence of forms $J_{r} \in \mathrm{M}_{2^{r}}(R)$ by the recurrence formulae:

$$
J_{r}= \begin{cases}1 & \text { for } r=0, \\ J_{r-1} \perp-J_{r-1}, & \text { for } r \text { even, } \\ J_{r-1} \top-J_{r-1}, & \text { for } r \text { odd. }\end{cases}
$$

$\left(\right.$ Here $\alpha \perp \beta=\left(\begin{array}{cc}\alpha & 0 \\ 0 & \beta\end{array}\right)$, while $\alpha \top \beta=\left(\begin{array}{cc}0 & \alpha \\ \beta & 0\end{array}\right)$.)

It is easy to see that $\operatorname{det}\left(J_{r}\right)=1$, for all $r$, and that $J_{r}^{T}=J_{r}^{-1}=(-1)^{\frac{r(r+1)}{2}} J_{r}$. Moreover, $J_{r}$ is antisymmetric if $r=4 k+1$ and $r=4 k+2$, whereas $J_{r}$ is symmetric for $r=4 k$ and $r=4 k+3$. In ([18, Lemma 5.3), it is noted that the following formulae are valid: The Suslin identities are

$$
\begin{aligned}
\text { for } r=4 k:\left(S_{r}(v, w) J_{r}\right)^{T} & =S_{r}(v, w) J_{r} \\
\text { for } r=4 k+1: S_{r}(v, w) J_{r} S_{r}(v, w)^{T} & =\left(v \cdot w^{T}\right) J_{r} \\
\text { for } r=4 k+2:\left(S_{r}(v, w) J_{r}\right)^{T} & =-S_{r}(v, w) J_{r} \\
\text { for } r=4 k+3: S_{r}(v, w) J_{r} S_{r}(v, w)^{T} & =\left(v \cdot w^{T}\right) J_{r} .
\end{aligned}
$$

Remark 2.11. Consider the Suslin matrix $S_{r}(v, w)$, with $\langle v, w\rangle=1$. By the Suslin identities, this is a symplectic matrix w.r.t. $J_{r}$ when $r \equiv 1$ modulo (4) and an orthogonal matrix w.r.t. $J_{r}$ when $r \equiv 3$ modulo (4).

Lemma 2.13 is the relative symplectic analogue of (15], Corollary 4.3). Before this we recall the excision ring $(\mathbb{Z} \oplus I)$ considered by W. van der Kallen in [12.

The excision ring $(\mathbb{Z} \oplus I)$. If $I$ is an ideal in $R$, one can then construct the ring $\mathbb{Z} \oplus I$ with multiplication defined by

$$
(n \oplus i)(m \oplus j)=(n m \oplus n j+m i+i j),
$$

for $m, n \in \mathbb{Z}, i, j \in I$.

The maximal spectrum of the ring $\mathbb{Z} \oplus I$ is noetherian, being the union of finitely many subspaces of dimension $\leq \operatorname{dim}(R)$.

There is a natural homomorphism $\varphi: \mathbb{Z} \oplus I \longrightarrow R$ given by $(m \oplus i) \mapsto m+i \in R$.

Next recall the lemma which enables one to reduce to the relative case in [15]:

Lemma 2.12 ([15, Lemma 4.2]). Let $R$ be a ring, and let $J$ be an ideal of $R$. Let $\alpha \in \mathrm{SL}_{n}(R)$ with $\alpha \equiv I_{n}$ modulo $J$. Then there is a unique matrix $S \in \mathrm{SL}_{n}(\mathbb{Z} \oplus J)$ such that $S \equiv I_{n}$ modulo $(0 \oplus J)$, and with $\varphi(S)=\alpha$, where $\varphi: \mathbb{Z} \oplus J \longrightarrow R$ is the natural homomorphism.

Moreover, if $n=2^{m}$, for some $m$, and $\alpha$ is a Suslin matrix of determinant one, then $S$ is also a Suslin matrix of determinant one. 
We shall follow a process of "cutting down of size" of the symplectic form $J_{r}$ below; i.e. we shall look at the forms which are obtained successively from $J_{r}$ by deleting their $i$-th and $\pi(i)$-th rows and columns, where $\pi$ is the underlying permutation of $J_{r}$. For simplicity, we shall just write $J_{r}$ for these forms, without referring to their size, as we are really considering the symplectic group of these forms sitting inside the symplectic group of $J_{r}$.

Lemma 2.13. Let $R$ be a commutative ring of dimension $d$, and let $I$ be an ideal of $R$. Let $S_{r}(v, w) \in \mathrm{SL}_{2^{r}}(R, I)$, for $v, w \in \mathrm{Um}_{r+1}(R, I)$, and with $\langle v, w\rangle=1$. Assume that $r \geq d$. Further assume that $r \equiv 1(\bmod 4)$.

Then there exists $\varepsilon_{J_{r}} \in \operatorname{ESp}\left(J_{r}\right)$ such that $S_{r}(v, w) \varepsilon_{J_{r}}=I_{k} \perp \gamma$, for some $\gamma \in \operatorname{Sp}_{J}(R, I)$ and for suitable $k$. Moreover, $\gamma$ and $\beta_{r}(v, w)$ are stably elementarily equivalent.

Proof. Consider the corresponding Suslin matrix $S_{r}\left(v^{*}, w^{*}\right) \in \mathrm{SL}_{2^{r}}(\mathbb{Z} \oplus I)$ described in Lemma 2.12. As $r \equiv 1(\bmod 4), S_{r}\left(v^{*}, w^{*}\right) \in \operatorname{Sp}_{J_{r}}(\mathbb{Z} \oplus I)$.

There is a $\beta_{r}\left(v^{*}, w^{*}\right) \in S_{r}\left(v^{*}, w^{*}\right) \mathrm{E}_{2^{r}}(\mathbb{Z} \oplus I)$, with $e_{1} \beta_{r}\left(v^{*}, w^{*}\right)=\chi_{r !}\left(\left[v^{*}\right]\right)$, and with $\beta_{r}\left(v^{*}, w^{*}\right) \equiv I_{r+1}$, modulo $I$. This follows from ([15], Corollary 4.3).

Since the dimension of the maximal spectrum of $(\mathbb{Z} \oplus I)$ is $d, \operatorname{Um}_{r}(\mathbb{Z} \oplus I)=$ $e_{1} \mathrm{E}_{r}(\mathbb{Z} \oplus I)$, for $r \geq \max \{4, d+2\}$. By Proposition 2.2

$$
\mathrm{Um}_{q}(\mathbb{Z} \oplus I,(0 \oplus I))=e_{1} \mathrm{E}_{q}(\mathbb{Z} \oplus I,(0 \oplus I)),
$$

for all $q \geq \max \{4, d+2\}$. By Theorem 2.8 ,

$$
\mathrm{Um}_{q}(\mathbb{Z} \oplus I,(0 \oplus I))=e_{1} \operatorname{ESp}_{q}(\mathbb{Z} \oplus I,(0 \oplus I)),
$$

for all $q \geq \max \{4, d+2\}$. (By Remark 2.9 we may take this to be the elementary symplectic group w.r.t. the symplectic form $J_{r}$.)

Let $e_{1} S_{r}\left(v^{*}, w^{*}\right)=e_{1} \varepsilon_{1}$, for some $\varepsilon_{1} \in \operatorname{ESp}_{J_{r}}(\mathbb{Z} \oplus I,(0 \oplus I))$. Then one has

$$
S_{r}\left(v^{*}, w^{*}\right) \varepsilon_{1}^{-1} \in \operatorname{Sp}_{J_{r}}(\mathbb{Z} \oplus I),
$$

whence is equal, after a suitable modification by an element of $\operatorname{ESp}_{J_{r}}(R)$, to $1 \perp S^{\prime}$, for some $S^{\prime}$, which also has its $\pi(1)$-row and $\pi(1)$-column to be $e_{\pi(1)}$, respectively $e_{\pi(1)}^{t}$, where $\pi$ denotes the underlying permutation of $J_{r}$. Let us rename the matrix obtained by removing this row and column and loosely write $S_{r}\left(v^{*}, w^{*}\right) \varepsilon_{1}^{-1}=\mathrm{I}_{2} \perp S$ for suitable $S$ derived from $S^{\prime}$ by removing a suitable row and column from it. This $S \in \operatorname{Sp}_{J_{r}}(\mathbb{Z} \oplus I)$, with the aforementioned convention.

Continue the above process with $S$ in place of $S_{r}\left(v^{*}, w^{*}\right)$ above and with the corresponding "sub form" of $J_{r}$ obtained by deleting the 1-st and $\pi(1)$-th rows and columns, etc. Note that since $S_{r}\left(v^{*}, w^{*}\right) \in \operatorname{Sp}_{J_{r}}(\mathbb{Z} \oplus I)$, it follows that $\gamma \in$ $\operatorname{Sp}_{J_{r}}(\mathbb{Z} \oplus I,(0 \oplus I))$. The first assertion over the ring $(\mathbb{Z} \oplus I)$ follows.

Now use the natural homomorphism $(\mathbb{Z} \oplus I) \longrightarrow R$ to deduce the first assertion over any ring $R$. Thus $S_{r}(v, w)$ contains a matrix $\gamma$ of size $(r+1)$ in its elementary symplectic class.

Since $\gamma$ is stably elementarily equivalent to $S_{r}(v, w)$, it follows that $\gamma$ and $\beta_{r}(v, w)$ are stably elementarily equivalent, as both are stably elementarily equivalent to $S_{r}(v, w)$.

2.3. Nice group structures. In the papers [12, 13] W. van der Kallen shows that the orbit space of unimodular rows modulo the elementary action $\operatorname{Um}_{n}(A) / \mathrm{E}_{n}(A)$ has an abelian group structure if $2 \leq d \leq 2 n-4$, where $d$ is the stable dimension of 
$A$. In fact he defines the notion of a weak Mennicke $n$-symbol in [13] and shows that the orbit space is bijective to the universal weak Mennicke $n$-symbol $\mathrm{WMS}_{n}(A)$, under the above conditions.

In 7 such groups were called nice if they satisfied 'coordinate-wise multiplication", viz. if in this group,

$$
\left[\left(a_{1}, a_{2}, \ldots, a_{n}\right)\right] *\left[\left(b_{1}, a_{2}, \ldots, a_{n}\right)\right]=\left[\left(a_{1} b_{1}, a_{2}, \ldots, a_{n}\right)\right] .
$$

The group structure is nice when

(a) (7], Theorem 3.9) $A$ is an affine algebra of dimension $d$ over a field $k$ of cohomological dimension at most one (c.d.2 $\leq 1$ suffices) and $n=d+1$. This result has been reproved in ([11, Proposition 3.5) by a different method when $k$ is algebraically closed.

(b) ([11, Proposition 3.5) $A$ is an affine algebra of dimension $d$ over the algebraic closure of a finite field $k$ (whose characteristic is not 2) and $n=d$.

(c) ([7], Theorem 5.1) $A=R[X]$, where $R$ is a noetherian local ring of dimension $d$, in which 2 is invertible, and $n=d+1$.

In case the group structure is nice, then in ([7, Theorem 2.1) it is shown that the universal weak Mennicke symbol is isomorphic to the universal Mennicke symbol.

In ( 7 , Corollary 2.2) it is deduced from this that if $A$ is a commutative ring of stable dimension $d$, then the first row $e_{1} \sigma$ of any stably elementary $\sigma \in \mathrm{SL}_{d+1}(A)$ can be completed to an elementary matrix.

\section{THE MAIN THEOREMS}

We are now ready to prove the main theorems stated in the introduction. We begin with

Proposition 3.1. Let $R$ be a commutative ring of dimension $d>1$, and let $I$ be an ideal of $R$. Assume that $d \equiv 1$ modulo (4). Let $v \in \operatorname{Um}_{d+1}(R, I)$. If $\operatorname{Um}_{d+1}(R, I) / \mathrm{E}_{d+1}(R, I)$ has a nice group structure, then $\chi_{d !}([v])$ can be completed to a symplectic matrix which is congruent to the identity modulo $I$.

Proof. Choose $w \in \operatorname{Um}_{d+1}(R, I)$ such that $\langle v, w\rangle=1$. By Lemma 2.13 there is a $\gamma \in \operatorname{Sp}_{d+1}(R, I)$ such that $\gamma$ and $\beta_{d}(v, w)$ are stably elementarily equivalent. By Proposition 2.2, $\left(1 \perp \beta_{d}(v, w) \gamma^{-1}\right) \in \mathrm{E}_{d+2}(R, I)$.

By ([13], Theorem 2.2) there is an $\varepsilon \in \mathrm{E}_{d+1}(R, I)$ such that

$$
\beta_{d}(v, w) \gamma^{-1} \varepsilon=\left(\begin{array}{cc}
1+t x & t y \\
z^{T} & M
\end{array}\right),
$$

for some $t, x \in R, y, z \in \mathrm{M}_{1, d}(R) . M \in \mathrm{M}_{d}(R)$, with $\left(\begin{array}{cc}1+t x & t y \\ z^{T} & M\end{array}\right) \in \mathrm{E}_{d+1}(R, I)$. Since the group structure on the orbit space $\operatorname{Um}_{d+1}(R, I) / \mathrm{E}_{d+1}(R, I)$ is nice,

$$
[(1+t x, t y)]=[(1+t x, y)]=1 .
$$

By Theorem 2.8, $[(1+t x, t y)]=[(1+t x, y)]=1$ in $\operatorname{Um}_{d+1}(R, I) / \operatorname{ESp}_{d+1}(R, I)$.

Hence, $e_{1} \beta_{d}(v, w)=e_{1} \gamma^{-1} \varepsilon_{0}$, for some $\varepsilon_{0} \in \operatorname{ESp}_{d+1}(R, I)$, and with $\gamma \in \operatorname{Sp}_{d+1}(R, I)$. The result follows. 
As a consequence of Proposition 3.1 we get Theorem 2:

Theorem 3.2. Let $R$ be an affine algebra of dimension $d>1$ over an infinite field of cohomological dimension at most one, and let $I=(f)$ be a principal ideal of $R$. Assume that $d \equiv 1$ modulo (4). Let $v \in \mathrm{Um}_{d+1}(R, I)$. Then $v$ can be completed to a symplectic matrix which is congruent to the identity modulo $I$.

Proof. By ([15], Proposition 5.4), $[v]=\left[\chi_{d !}\left(\left[v^{\prime}\right]\right)\right]$ in $\operatorname{Um}_{d+1}(R, I) / \mathrm{E}_{d+1}(R, I)$.

By Theorem 2.8, $[v]=\left[\chi_{d !}\left(\left[v^{\prime}\right]\right)\right]$ in $\operatorname{Um}_{d+1}(R, I) / \operatorname{ESp}_{d+1}(R, I)$.

Work with $v^{\prime *}$ (the vector corresponding to $v^{\prime}$ ) over the excision algebra $B$ w.r.t. $I$ as was done in Lemma 2.10 .

Now apply Proposition 3.1 to $v^{\prime *} \in \operatorname{Um}_{d+1}(B,(Z)) / \mathrm{E}_{d+1}(B,(Z))$ to deduce that it can be completed to a symplectic matrix which is congruent to the identity modulo $(Z)$. Now come back to $A$ and deduce the result for $v$.

We can now establish Theorem 1:

Without loss of generality we may assume that $2 n=d$. Let $\sigma \in \operatorname{Sp}_{d}(A) \cap$ $\operatorname{ESp}_{d+2}(A)$. Let $\delta(T) \in \operatorname{ESp}_{d+2}(A[T])$ be a symplectic homotopy between $\mathrm{I}_{d+2}$ and $\mathrm{I}_{2} \perp \sigma$, i.e. $\delta(1)=\mathrm{I}_{2} \perp \sigma$ and $\delta(0)=\mathrm{I}_{d+2}$. Let $v(T)=e_{1} \delta(T) \in \operatorname{Um}_{d+2}(A[T])$.

By Theorem 3.2, applied to $v(T)$, and w.r.t. the principal ideal $I=\left(T^{2}-T\right)$, we get $v(T)=e_{1} \alpha(T)$, for some $\alpha(T) \in \operatorname{Sp}_{d+2}\left(A[T],\left(T^{2}-T\right)\right)$. Now

$$
\delta(T) \alpha(T)^{-1}=\left(\begin{array}{ccc}
1 & 0 & 0 \\
* & 1 & * \\
* & 0 & \eta(T)
\end{array}\right),
$$

for some $\eta(T) \in \operatorname{Sp}_{d}(A[T])$. Clearly, $\eta(T)$ is a symplectic homotopy of $\sigma$.

If $A$ is non-singular, then by ([4], Theorem 3.8), $\operatorname{Sp}_{d}\left(A_{\mathfrak{p}}[T]\right)=\operatorname{ESp}_{d}\left(A_{\mathfrak{p}}[T]\right)$, for all $\mathfrak{p} \in \operatorname{Spec}(A)$. Consequently, $\eta(T) \in \operatorname{ESp}_{d}(A[T])$. Hence, $\sigma=\eta(1) \in$ $\operatorname{ESp}_{d}(A)$.

\section{REFERENCES}

[1] H. Bass, Algebraic K-theory, Benjamin, New York, 1968. MR0249491 (40:2736)

[2] H. Bass, J. Milnor, J-P. Serre, Solution of the congruence subgroup problem for $\mathrm{SL}_{n}, n \geq 3$ and $\mathrm{Sp}_{2 n}, n \geq 2$, Inst. Publ. Math. IHES 33 (1967), 59-137. MR0244257(39:5574)

[3] H. Bass, K-theory and stable algebra, Inst. Publ. Math. IHES 22 (1964), 5-60. MR0174604 $(30: 4805)$

[4] R. Basu, R.A. Rao, Injective stability for $K_{1}$ of classical modules, Journal of Algebra 323 (2010), 867-877. MR2578583

[5] R. Basu, Ravi A. Rao, Selby Jose, Injective stability for $K_{1}$ of the orthogonal group, Journal of Algebra 323 (2010), 393-396. MR2564846

[6] P. Chattopadhyay, R.A. Rao, Elementary symplectic orbits and improved $K_{1}$-stability, to appear in Journal of $K$-theory, doi:10.1017/is010002021jkt109, published online by Cambridge University Press, 10 June 2010.

[7] A. Garge, R.A. Rao, A nice group structure on the orbit space of unimodular rows. $K$-Theory 38 (2008), no. 2, 113-133. MR2366558 (2009f:19005)

[8] Jean Fasel, Stably free modules over smooth affine threefolds, arXiv:0911.3495v2 [math.AC], to appear in Duke Mathematical Journal.

[9] Selby Jose, Ravi A. Rao, A structure theorem for the elementary unimodular vector group. Trans. Amer. Math. Soc. 358 (2006), no. 7, 3097-3112. MR2216260 (2007a:20047)

[10] Selby Jose, Ravi A. Rao, A fundamental property of Suslin matrices, Journal of $K$-theory: $K$-theory and its Applications to Algebra, Geometry, and Topology 5 (2010), no. 3, 407-436.

[11] Ravi A. Rao, Selby Jose, A group structure on squares. Proc. Amer. Math. Soc. 136 (2008), no. 4, 1181-1191. MR2367092 (2008j:13016)

[12] W. van der Kallen, A group structure on certain orbit sets of unimodular rows, Journal of Algebra 82 (1983), no. 2, 363-397. MR704762 (85b:13014) 
[13] W. van der Kallen, A module structure on certain orbit sets of unimodular rows, J. Pure and Appl. Algebra 57 (1989), 281-316. MR.987316 (90b:18013)

[14] R.A. Rao, W. van der Kallen, Improved stability for $S K_{1}$ and $W M S_{d}$ of a non-singular affine algebra. $K$-theory (Strasbourg, 1992). Astérisque 226 (1994), 411-420. MR.1317126 (96e:19001)

[15] Ravi A. Rao, A stably elementary homotopy. Proc. Amer. Math. Soc. 137 (2009), no. 11, 3637-3645. MR 2529870 (2010h:19002)

[16] Ravi A. Rao, Rabeya Basu, Selby Jose, Injective stability for $K_{1}$ of the orthogonal group. Journal of Algebra, 323 (2010), no. 2, 393-396. MR2564846

[17] A.A. Suslin, L.N. Vaserstein, Serre's problem on projective modules over polynomial rings and algebraic $K$-theory, Math. USSR Izvestija 10 (1976), 937-1001. MR0447245 (56:5560)

[18] A.A. Suslin, Stably Free Modules. (Russian) Math. USSR Sbornik 102 (144) (1977), no. 4, 537-550. Mat. Inst. Steklov. (LOMI) 114 (1982), 187-195. MR0441949 (56:340)

[19] A.A. Suslin, On the structure of the special linear group over polynomial rings, Math. USSR. Izvestija 11 (1977), 221-238. MR0472792 (57:12482)

[20] A.A. Suslin, Mennicke symbols and their applications in the $K$-theory of fields. Algebraic $K$ theory, Part I (Oberwolfach, 1980), 334-356, Lecture Notes in Math., 966, Springer, BerlinNew York, 1982. MR689382 (84f:18023)

[21] A.A. Suslin, The cancellation problem for projective modules, and related questions. (Russian) Proceedings of the International Congress of Mathematicians (Helsinki, 1978), 323-330, Acad. Sci. Fennica, Helsinki, 1980. MR562623 (83e:13011)

[22] A.A. Suslin, Cancellation for affine varieties. (Russian) Modules and algebraic groups. Zap. Nauchn. Sem. Leningrad. Otdel. Mat. Inst. Steklov. (LOMI) 114 (1982), 187-195, 222. MR669571(84c:14012)

[23] L.N. Vaserstein, On the stabilization of the general linear group over a ring, Mat. Sbornik (N.S.) 79 (121), 405-424 (Russian); English translation in Math. USSR-Sbornik 8 (1969), 383-400. MR0267009 (42:1911)

[24] L.N. Vaserstein, Stabilization of unitary and orthogonal groups over a ring with involution, Mat. Sbornik 81 (123), no. 3 (1970), 328-351. MR0269722 (42:4617)

[25] L.N. Vaserstein, Operations on orbits of unimodular vectors. Journal of Algebra 100 (1986), no. 2, 456-461. MR840588 (87j:13018)

Indian Institute of Science Education and Research, Kolkata 741 252, India

E-mail address: rbasu@iiserkol.ac.in

Institute of Mathematical Sciences, Chennai 600 113, India

E-mail address: pratyusha@imsc.res.in

Tata Institute of Fundamental Research, Mumbai 400 005, India

E-mail address: ravi@math.tifr.res.in 